TRANSACTIONS OF THE

AMERICAN MATHEMATICAL SOCIETY

Volume 355 , Number 12, Pages 4807-4823

S 0002-9947(03)03042-3

Article electronically published on July 24, 2003

\title{
A LOCAL CHARACTERIZATION OF SIMPLY-LACED CRYSTALS
}

\author{
JOHN R. STEMBRIDGE
}

\begin{abstract}
We provide a simple list of axioms that characterize the crystal graphs of integrable highest weight modules for simply-laced quantum KacMoody algebras.
\end{abstract}

\section{INTRODUCTION}

Following Kashiwara [K2], a crystal is an edge-colored directed graph satisfying a simple set of axioms. Every (integrable highest weight) representation of a symmetrizable quantum Kac-Moody algebra has a crystal associated to it that encodes important combinatorial data. For example, knowing the crystal of a representation allows one to immediately deduce tensor product and branching rules involving that representation. There are a number of explicit constructions known for the crystals of representations of particular quantum algebras; e.g., [KN], [L3]. In the cases of finite type (i.e., quantum enveloping algebras of semisimple Lie algebras), it is also possible to give explicit descriptions of crystals in terms of the integer points of a convex polytope [BZ, [L4]. In the general case, Littelmann's Path Model [L1], L2] provides an algorithm to generate the crystal of any representation.

On the other hand, the crystals of representations form a very special subclass of the set of all crystals, and it has been an open problem to find a simple set of local axioms that characterize them. In other words, can one determine from local structural conditions whether a crystal graph is the crystal of a representation? In this paper, we give an affirmative solution in the simply-laced cases; i.e., for quantum Kac-Moody algebras with a Cartan matrix whose off-diagonal entries are 0 or -1 . These simply-laced crystals are arguably the most important, since all highest weight crystals of finite or affine type - the ones of widest interest - are either simply-laced or may be obtained from such crystals by a standard technique of "folding" by diagram automorphisms.

Among the properties we use to characterize these crystals, (P1)-(P3) are equivalent to the defining axioms for a general crystal (aside from the fact that we have not explicitly required the assignment of weight vectors to the vertices). The key additional axioms are two relations $(\mathrm{P} 5)-(\mathrm{P} 6)$ and their duals $\left(\mathrm{P} 5^{\prime}\right)-\left(\mathrm{P} 6^{\prime}\right)$ that may be viewed as combinatorial analogues of the Serre relations. Roughly speaking, they require that for each (distinct) pair of raising operators $E_{i}$ and $E_{j}$, one

Received by the editors February 12, 2002.

2000 Mathematics Subject Classification. Primary 17B37, 05 E99.

This research was partially supported by NSF Grant DMS-0070685 and the Guggenheim Foundation. 
of the relations

$$
E_{i} E_{j}(x)=E_{j} E_{i}(x) \quad \text { or } \quad E_{i} E_{j}^{2} E_{i}(x)=E_{j} E_{i}^{2} E_{j}(x)
$$

holds whenever $E_{i}(x)$ and $E_{j}(x)$ are defined, and furthermore, one may use data localized at $x$ to determine which of these two relations must hold. The analogous dual relations involve the lowering operators $F_{i}$ and $F_{j}$.

We have used relations very similar to these in Section 6 of [St], although the context there is limited to the crystals for the cases of finite type, and we did not attempt to formulate the relations in a way that uniquely characterized the crystals. It is also interesting to note that these relations are similar to some relations due to Kashiwara (see Section 7.3 of [K1]); for example, if $i$ and $j$ index a subsystem of type $A_{2}$, then

$$
E_{i}^{a} E_{j}^{a+b} E_{i}^{b}(x)=E_{j}^{b} E_{i}^{a+b} E_{j}^{a}(x) \quad(a, b \geqslant 0)
$$

whenever both sides are defined. Relations such as these are not equivalent to our axioms, since they do not tell us a priori whether the expressions are defined. Another distinction is that the properties we describe in (P5)-(P6) are valid even in the non-simply-laced cases, and do not depend on the entries of the Cartan matrix. In fact, the Cartan matrix appears only once among the axioms, in (P3).

The paper is organized as follows. In Section 1 we present the axioms, and prove that they admit at most one model (up to isomorphism) for each choice of highest weight. We also describe a simple algorithm for generating these models (see Remark 1.5). In Section 2, we prove that Littelmann's path operators generate graphs that satisfy the axioms; since the Path Model is known to produce the crystal graphs of irreducible highest weight representations, this allows us to conclude in Section 3 that our axioms characterize the crystals of highest weight modules in the simply-laced case (Theorem 3.3). Also in Section 3, we discuss some of the obstacles that need to be overcome in order to generalize our results beyond the simply-laced case.

We remark that by Proposition 2.4.4 of [KMN], it is known that a crystal with a unique maximal vertex is the crystal of a highest weight representation if and only if it decomposes as a disjoint union of such crystals relative to the rank 2 subalgebras corresponding to each pair of edge colors. Thus, an alternative (and shorter) approach to our main result would be to prove that the claimed relations hold for crystals of type $A_{2}$ and $A_{1} \times A_{1}$. Moreover, this is the essential content of Lemmas 6.5 and 6.6 in St. However, it should be emphasized that what we prove here using the Path Model goes beyond the simply-laced case. Indeed, we prove that the relations hold in all cases, simply-laced or not. The key difference is that only in the simply-laced cases are these relations strong enough to provide a unique characterization.

\section{A-Regular posets}

Let $I$ be a finite index set and $A=\left[a_{i j}\right]_{i, j \in I}$ the Cartan matrix of a simply-laced Kac-Moody algebra; i.e., a matrix for which $a_{i i}=2$ and $a_{i j}=a_{j i} \in\{0,-1\}$ for all $i \neq j$.

We seek to associate to $A$ a class of (possibly infinite) edge-colored directed graphs that we call $A$-regular. By "edge-colored," we mean that each edge has been assigned a label from the index set $I$. Given such a graph $X$, our first requirements for $A$-regularity follow. 
(P1) All monochromatic directed paths in $X$ have finite length. In particular, $X$ has no monochromatic circuits.

(P2) For every vertex $x$ and every $i \in I$, there is at most one edge $x \leftarrow y$ with color $i$, and dually, at most one edge $x \rightarrow z$ with color $i$.

We define $y=E_{i}(x)$ if there is an $i$-colored edge $x \leftarrow y$, and dually $z=F_{i}(x)$ if there is an $i$-colored edge $x \rightarrow z$. In this way, $E_{i}$ and $F_{i}$ form an inverse pair of (partial) operators on $X$. Note that $F_{i}$ follows the orientation of $X$, whereas $E_{i}$ follows the orientation of the reversed graph $X^{*}$.

We define the $i$-string through $x$ to be the (finite, by (P1)) maximal path of the form

$$
F_{i}^{-d}(x) \rightarrow \cdots \rightarrow F_{i}^{-1}(x) \rightarrow x \rightarrow F_{i}(x) \rightarrow \cdots \rightarrow F_{i}^{r}(x) \quad(r, d \geqslant 0) .
$$

The $i$-rise and $i$-depth of $x$ are defined to be $\varepsilon(x, i):=r$ and $\delta(x, i):=-d$, respectively. Thus, $F_{i}(x)$ is defined if and only if $\varepsilon(x, i)>0$, and $E_{i}(x)$ is defined if and only if $\delta(x, i)<0$.

In order to measure the effect of the operators $E_{i}$ and $F_{i}$ on the $j$-depth and $j$-rise of each vertex, let us define difference operators $\Delta_{i}$ so that

$$
\Delta_{i} \delta(x, j)=\delta\left(E_{i} x, j\right)-\delta(x, j), \quad \Delta_{i} \varepsilon(x, j)=\varepsilon\left(E_{i} x, j\right)-\varepsilon(x, j)
$$

whenever $E_{i} x$ is defined (i.e., $\delta(x, i)<0$ ), and dually

$$
\nabla_{i} \delta(x, j)=\delta(x, j)-\delta\left(F_{i} x, j\right), \quad \nabla_{i} \varepsilon(x, j)=\varepsilon(x, j)-\varepsilon\left(F_{i} x, j\right)
$$

whenever $F_{i} x$ is defined (i.e., $\varepsilon(x, i)>0$ ). Note that in the reversed graph $X^{*}$, the roles of $E_{i}, \varepsilon, \delta, \Delta_{i} \varepsilon$ and $\Delta_{i} \delta$ are interchanged with $F_{i},-\delta,-\varepsilon, \nabla_{i} \delta$, and $\nabla_{i} \varepsilon$.

Now fix $x \in X$ and a distinct pair $i, j \in I$. Assuming $E_{i} x$ is defined, we require

(P3) $\Delta_{i} \delta(x, j)+\Delta_{i} \varepsilon(x, j)=a_{i j}$, and

(P4) $\Delta_{i} \delta(x, j) \leqslant 0, \Delta_{i} \varepsilon(x, j) \leqslant 0$.

Note that (P3) is valid even for $i=j$, since it is clear from the definition that the $i$-depth and $i$-rise both increase by 1 in passing from $x$ to $E_{i} x$.

We remark that since $a_{i j} \in\{0,-1\},(\mathrm{P} 3)$ and (P4) allow for only three possibilities:

$$
\left(a_{i j}, \Delta_{i} \delta(x, j), \Delta_{i} \varepsilon(x, j)\right)=(0,0,0),(-1,-1,0), \text { or }(-1,0,-1) .
$$

These are illustrated in Figure 1.

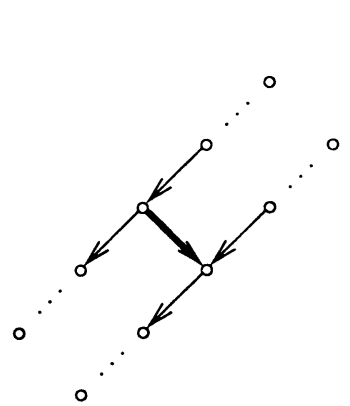

(a) $a_{i j}=0$

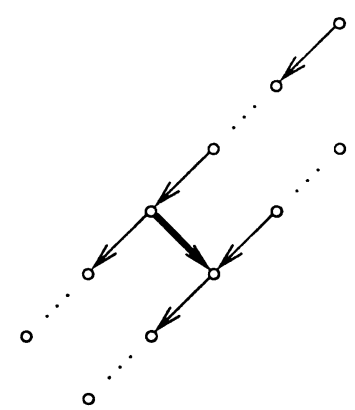

(b) $a_{i j}=\Delta_{i} \delta(x, j)=-1$

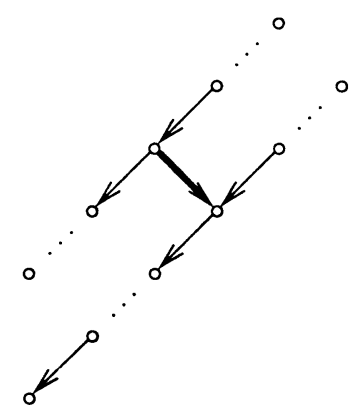

(c) $a_{i j}=\Delta_{i} \varepsilon(x, j)=-1$

FIgURE 1. The effect of $E_{i}$ on $j$-strings ( $i=$ thick, $j=$ thin). 

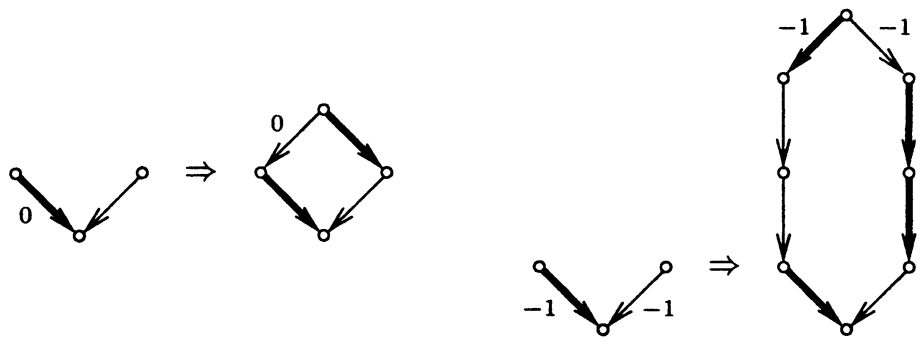

Figure 2. Axioms (P5) and (P6).
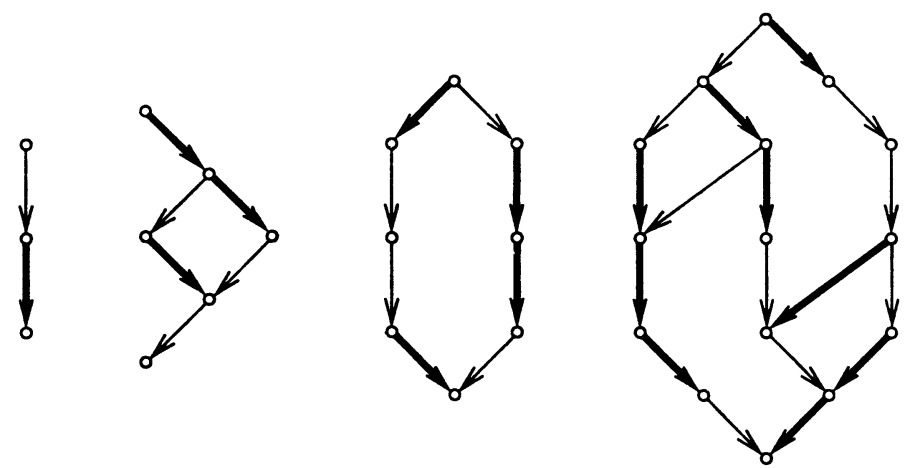

FiguRE 3. $A$-regular graphs for the Cartan matrix $A=\left[\begin{array}{rr}2 & -1 \\ -1 & 2\end{array}\right]$.

Now assuming that $E_{i} x$ and $E_{j} x$ are both defined, we require that:

(P5) $\Delta_{i} \delta(x, j)=0$ implies $E_{i} E_{j} x=E_{j} E_{i} x$ and $\nabla_{j} \varepsilon(y, i)=0$, where $y=$ $E_{i} E_{j} x=E_{j} E_{i} x$

(P6) $\Delta_{i} \delta(x, j)=\Delta_{j} \delta(x, i)=-1$ implies $E_{i} E_{j}^{2} E_{i} x=E_{j} E_{i}^{2} E_{j} x$ and $\nabla_{i} \varepsilon(y, j)=$ $\nabla_{j} \varepsilon(y, i)=-1$, where $y=E_{i} E_{j}^{2} E_{i} x=E_{j} E_{i}^{2} E_{j} x$.

These axioms are illustrated in Figure 2. (In the figures, a label below an $i$-colored edge $x \leftarrow y$ indicates the value of $\Delta_{i} \delta(x, j)$; a label above indicates $\nabla_{i} \varepsilon(y, j)$.)

We remark that $\delta\left(E_{i} x, j\right) \leqslant \delta(x, j)<0$ by (P4) (and similarly with $i$ and $j$ interchanged); so the expressions $E_{j} E_{i} x$ and $E_{i} E_{j} x$ appearing in (P5) are defined. On the other hand, while it is easy to show that the hypothesis of (P6) forces $E_{j}^{2} E_{i} x$ and $E_{i}^{2} E_{j} x$ to be defined, it is not obvious that the same is true for $E_{i} E_{j}^{2} E_{i} x$ and $E_{j} E_{i}^{2} E_{j} x$; so this should be considered implicit in (P6).

Dually, when $F_{i} x$ and $F_{j} x$ are defined, we also require that:

$\left(\mathrm{P} 5^{\prime}\right) \nabla_{i} \varepsilon(x, j)=0$ implies $F_{i} F_{j} x=F_{j} F_{i} x$ and $\Delta_{j} \delta(y, i)=0$, where $y=F_{i} F_{j} x=$ $F_{j} F_{i} x$.

$\left(\mathrm{P} 6^{\prime}\right) \nabla_{i} \varepsilon(x, j)=\nabla_{j} \varepsilon(x, i)=-1$ implies $F_{i} F_{j}^{2} F_{i} x=F_{j} F_{i}^{2} F_{j} x$ and $\Delta_{i} \delta(y, j)=$ $\Delta_{j} \delta(y, i)=-1$, where $y=F_{i} F_{j}^{2} F_{i} x=F_{j} F_{i}^{2} F_{j} x$.

Definition 1.1. Let $A$ be a simply-laced Cartan matrix. An edge-colored directed graph is $A$-regular if it satisfies $(\mathrm{P} 1)-(\mathrm{P} 6)$ and $\left(\mathrm{P} 5^{\prime}\right)-\left(\mathrm{P} 6^{\prime}\right)$.

Some examples of $A$-regular directed graphs appear in Figure 3. 
It is easy to see that $X$ is $A$-regular if and only if every (weak) connected component of $X$ is $A$-regular, so there is no harm in restricting our attention to the connected case.

A vertex $x \in X$ is maximal if there are no edges $x \leftarrow y$; i.e., $\delta(x, i)=0$ for all $i \in I$.

Proposition 1.2. A connected A-regular directed graph has a maximal vertex if and only if it is the Hasse diagram of a ranked poset with a maximum element.

Proof. Given an $A$-regular graph $X$ with a maximal vertex $x_{0}$, define $x \leqslant y$ if there is a directed path from $y$ to $x$. If there is a pair $x \leqslant y$ in $X$ such that $x \leqslant x_{0}$ and $y \nless x_{0}$, then among all such pairs, choose one that minimizes the length of a directed path from $x_{0}$ to $x$. Clearly, $x \neq x_{0}$, so we have $x \leftarrow E_{i} x \leqslant x_{0}$ and $E_{j} x \leqslant y$ for some (distinct) pair $i, j \in I$. Since (P3) and (P4) imply $\Delta_{i} \delta(x, j), \Delta_{j} \delta(x, i) \in\{0,-1\}$, we may apply either (P5) or (P6) (possibly with $i$ and $j$ interchanged). In either case, we infer the existence of a vertex $z$ such that $E_{i} x \leqslant z$ and $y \leqslant z \nless x_{0}$, contradicting our choice of $x$. Thus the set of elements $\leqslant x_{0}$ is closed with respect to the action of both $E_{i}$ and $F_{i}$. Given that $X$ is connected, it follows that $x \leqslant x_{0}$ for all $x$.

For each $x \in X$, we claim that the number of edges in a directed path from $x_{0}$ to $x$ does not depend on the choice of path. (Hence the graph is acyclic, and the relation " $\leqslant$ " is a ranked partial ordering.) Proceeding by induction, suppose that $l$ is the minimum length of a directed path from $x_{0}$ to $x$; we seek to show that every directed path from $x_{0}$ to $E_{i} x$ has length $l-1$ for all $i$ such that $E_{i} x$ is defined. There is at least one path of length $l-1$ from $x_{0}$ to some $E_{j} x$, whence all paths from $x_{0}$ to $E_{j} x$ have length $l-1$ by the induction hypothesis. For $i \neq j$, we may again use (P5) or (P6) to infer the existence of an element $y=E_{i} E_{j} x=E_{j} E_{i} x$ or $y=E_{i} E_{j}^{2} E_{i} x=E_{j} E_{i}^{2} E_{j} x$. Thus any directed path from $x_{0}$ to $y$ can reach $E_{i} x$ or $E_{j} x$ in the same number of steps; so by the induction hypothesis, every directed path from $x_{0}$ to $E_{i} x$ has length $l-1$ and the claim follows.

Remark 1.3. (a) The relations $E_{i} E_{j}=E_{j} E_{i}$ in (P5) and $E_{i} E_{j}^{2} E_{i}=E_{j} E_{i}^{2} E_{j}$ in (P6) are finely homogeneous, so the above reasoning may be used to show that every connected $A$-regular directed graph with a maximal vertex has a $\mathbf{Z}^{I}$-grading; i.e., the number of $i$-colored edges in a directed path from $y$ to $x$ is path-independent.

(b) An $A$-regular graph need not be acyclic. The simplest example is a 3 -cycle with one edge of each color, where $A$ is the $3 \times 3$ Cartan matrix with $a_{i j}=-1$ for all distinct $i, j$.

(c) If the Cartan matrix $A$ has an inverse $B=\left[b_{i j}\right]$, then every $A$-regular graph has a $\mathbf{Z}^{I}$-grading (and is therefore acyclic). Indeed, if we define

$$
\mu(x, j)=\sum_{i \in I} b_{i j}(\delta(x, i)+\varepsilon(x, i)),
$$

then (P3) implies $\mu\left(E_{i} x, j\right)-\mu(x, j)=\delta_{i j}$, and hence the number of $i$-colored edges in every directed path from $y$ to $x$ is $\mu(y, i)-\mu(x, i)$.

By an $A$-regular poset, we mean a (locally finite) partial ordering, together with an $A$-regular coloring of its covering edges.

Proposition 1.4. If $P$ and $P^{\prime}$ are A-regular posets with maximum elements $x$ and $x^{\prime}$, then $P$ and $P^{\prime}$ are isomorphic (as edge-colored digraphs) if and only if $\varepsilon(x, i)=\varepsilon\left(x^{\prime}, i\right)$ for all $i \in I$. Moreover, the isomorphism is unique. 
Proof. By Proposition 1.2, we know that $P$ and $P^{\prime}$ must be ranked. Proceeding by induction, assume that we have established the existence of a unique colorpreserving isomorphism between the portions of $P$ and $P^{\prime}$ above a given rank. We also assume (as part of the induction hypothesis) that this isomorphism preserves $\varepsilon$, even though the rise of an element may depend on parts of $P$ or $P^{\prime}$ below the rank in question. Given that $\varepsilon(x, i)=\varepsilon\left(x^{\prime}, i\right)$ for all $i \in I$, one may start the induction with the assignment $x \mapsto x^{\prime}$.

Now consider an element $y \in P$ and corresponding element $y^{\prime} \in P^{\prime}$ such that the ranks of $y$ and $y^{\prime}$ are at the lowest level where the current isomorphism is defined. If $F_{i} y$ exists, then $\varepsilon\left(y^{\prime}, i\right)=\varepsilon(y, i)>0$, and hence $F_{i} y^{\prime}$ also exists. Therefore, to extend the isomorphism to the next rank, we are forced to assign $F_{i} y \mapsto F_{i} y^{\prime}$. The main task then is to show that these new assignments are consistent; i.e., that $F_{i} y_{1}=F_{j} y_{2}$ implies $F_{i} y_{1}^{\prime}=F_{j} y_{2}^{\prime}$. This property and its converse (which follows by reasons of symmetry) allow us to conclude that the isomorphism extends uniquely to the next rank.

Thus let us suppose $z=F_{i} y_{1}=F_{j} y_{2}$, where $i, j \in I$ are distinct and $z \in P$ is an element of the next rank. If $\Delta_{i} \delta(z, j)=\Delta_{j} \delta(z, i)=-1$, one may use (P6) to infer the existence of an element $y \in P$ such that $y=E_{i} E_{j}^{2} E_{i} z=E_{j} E_{i}^{2} E_{j} z$ and $\nabla_{i} \varepsilon(y, j)=\nabla_{j} \varepsilon(y, i)=-1$. By the induction hypothesis, it follows that $\nabla_{i} \varepsilon\left(y^{\prime}, j\right)=\nabla_{j} \varepsilon\left(y^{\prime}, i\right)=-1$ in $P^{\prime}$, and hence $F_{i} y_{1}^{\prime}=F_{i} F_{j}^{2} F_{i} y^{\prime}=F_{j} F_{i}^{2} F_{j} y^{\prime}=F_{j} y_{2}^{\prime}$ by $\left(\mathrm{P} 6^{\prime}\right)$.

Since (P3) and (P4) imply that $\Delta_{i} \delta(z, j)$ and $\Delta_{j} \delta(z, i)$ are both 0 or -1 , the remaining possibility is that $\Delta_{i} \delta(z, j)=0$ or $\Delta_{j} \delta(z, i)=0$. Interchanging $i$ and $j$ if necessary, let us assume the former. This given, (P5) implies the existence of an element $y \in P$ such that $y=E_{i} E_{j} z=E_{j} E_{i} z$ and $\nabla_{j} \varepsilon(y, i)=0$. By the induction hypothesis, it follows that $\nabla_{j} \varepsilon\left(y^{\prime}, i\right)=0$, and therefore $F_{i} y_{1}^{\prime}=F_{i} F_{j} y^{\prime}=F_{j} F_{i} y^{\prime}=$ $F_{j} y_{2}^{\prime}$ by $\left(\mathrm{P} 5^{\prime}\right)$.

To complete the induction, it remains to be shown that this extension of the isomorphism still preserves $\varepsilon$. Given a corresponding pair of elements $z, z^{\prime}$ at the new rank, there must be an index $i \in I$ for which $E_{i} z$ (and hence also $E_{i} z^{\prime}$ ) is defined. By the induction hypothesis, it follows that $\varepsilon(z, i)=\varepsilon\left(E_{i} z, i\right)-1=$ $\varepsilon\left(E_{i} z^{\prime}, i\right)-1=\varepsilon\left(z^{\prime}, i\right)$. For the remaining indices $j \neq i$, note that the values of $\delta(z, j), \delta\left(E_{i} z, j\right)$ and $\varepsilon\left(E_{i} z, j\right)$ must match the corresponding values for $z^{\prime}$, the last of these via the induction hypothesis and the first two via the fact that $j$-depths depend only on the structure of $P$ or $P^{\prime}$ above the rank in question. However, (P3) shows that $\varepsilon(z, j)$ is a function of these three quantities, and hence is preserved by the isomorphism.

Remark 1.5. Given a nonnegative integer $I$-tuple, say $\mu=\left(\mu_{i}: i \in I\right)$, the above result shows that there is at most one $A$-regular poset $P(\mu)$ with a maximum element $x_{0}$ such that $\varepsilon\left(x_{0}, i\right)=\mu_{i}$ for $i \in I$. Moreover, a careful examination of the proof shows that if we assume the existence of $P(\mu)$, it can be constructed via the following algorithm.

The initial datum consists of the rise vector of the maximum element $x_{0}$. We then iteratively use the edges and rise vectors involving elements at or above a given rank $r$ to determine the corresponding data for the next lower rank:

A. For each pair $(x, i)$ such that $x$ has rank $r$ and $\varepsilon(x, i)>0$, create an element $y$ and an $i$-colored edge $x \rightarrow y$ (i.e., set $y=F_{i} x$ ). 
B. For each new pair of created elements, say $y=F_{i} x$ and $y^{\prime}=F_{j} x^{\prime}$, identify $y$ and $y^{\prime}$ if there is an element $z$ such that

(i) $z=E_{j} x=E_{i} x^{\prime}$ and $\nabla_{j} \varepsilon(z, i)=0$, or

(ii) $z=E_{i} E_{j}^{2} x=E_{j} E_{i}^{2} x^{\prime}$ and $\nabla_{i} \varepsilon(z, j)=\nabla_{j} \varepsilon(z, i)=-1$.

C. For each new element $y=F_{i} x$, determine the corresponding rise vector according to the rule $\varepsilon(y, j)=\varepsilon(x, j)+\delta(x, j)-\delta(y, j)-a_{i j}$ for all $j \in I$.

Certainly the above algorithm describes a method for constructing an edgecolored directed graph, but it is not at all obvious that the result is always $A$-regular. (Nor is it obvious that rule C produces well-defined "rise vectors" for each element that agree with the lengths of descending monochromatic paths.) Nevertheless, we will see that $A$-regular posets $P(\mu)$ exist for every choice of $\mu$, so this algorithm is necessarily valid.

The reader may find it instructive at this point to use the algorithm to reproduce the examples in Figure 3, starting only with the rise vectors of the maximum elements; namely, $\mu=(0,1),(2,0),(1,1)$ and $(1,2)$, respectively.

\section{2. $A$-Integrable SETS}

In this section, we discard the simply-laced hypothesis and allow $A$ to be any generalized Cartan matrix. That is, we assume only that $a_{i i}=2, a_{i j} \in \mathbf{Z} \leqslant 0$ (for $i \neq j)$, and $a_{i j}=0$ implies $a_{j i}=0$. Certainly the axioms (P1)-(P6) and (P5')$\left(\mathrm{P} 6^{\prime}\right)$ are meaningful in this more general context; however, the consequences of the axioms we derived in Section 1, such as the uniqueness result of Proposition 1.4, do require the simply-laced hypothesis.

Our goal here is to show that Littelmann's path operators produce $A$-regular posets. Unlike [L1], [L2], we are not assuming that $A$ is symmetrizable 1 so we need to take extra care in formulating the definitions.

Let $\mathcal{C}^{I}$ denote the space of all continuous maps $x:[0,1] \rightarrow \mathbf{R}^{I}$ with $x(0)=0$, where $\mathbf{R}^{I}$ denotes an $|I|$-dimensional real vector space. For all such $x$, we define

$$
\delta(x, i):=\min _{0 \leqslant t \leqslant 1} x_{i}(t), \quad \varepsilon(x, i):=\max _{0 \leqslant t \leqslant 1} x_{i}(1)-x_{i}(t) \quad(i \in I),
$$

where $x_{i}(t)$ denotes the $i$-th coordinate of $x$ at time $t$. Note that $\delta(x, i) \leqslant 0$, $\varepsilon(x, i) \geqslant 0$ and $\varepsilon(x, i)+\delta(x, i)=x_{i}(1)$. Eventually we will relate these quantities to the depth and rise of objects in an edge-colored directed graph, but in this general context, $\delta(x, i)$ and $\varepsilon(x, i)$ need not even be integer-valued.

For each subinterval $[b, c]$ of $[0,1]$ and each index $i \in I$, let us define a "reflection" operator $S_{i}=S_{i}(b, c)$ on $\mathcal{C}^{I}$ by setting

$$
\left(S_{i} x\right)_{j}(t)=\left\{\begin{array}{cc}
x_{j}(t) & \text { if } 0 \leqslant t \leqslant b \\
x_{j}(t)-a_{i j}\left(x_{i}(t)-x_{i}(b)\right) & \text { if } b \leqslant t \leqslant c \\
x_{j}(t)-a_{i j}\left(x_{i}(c)-x_{i}(b)\right) & \text { if } c \leqslant t \leqslant 1 .
\end{array}\right.
$$

These operators are closely related to the simple reflections $\left\{s_{i}: i \in I\right\}$ that generate the Weyl group associated to $A$; these are the linear operators on $\mathbf{R}^{I}$ defined by the rule

$$
\left(s_{i} u\right)_{j}=u_{j}-a_{i j} u_{i} \quad\left(u \in \mathbf{R}^{I}\right) .
$$

\footnotetext{
${ }^{1} \mathrm{~A}$ generalized Cartan matrix $A$ is symmetrizable if there is a (nonsingular) diagonal matrix $D$ such that $D A$ is symmetric. Simply-laced Cartan matrices are clearly symmetrizable.
} 
It is well known and easy to deduce from this rule that the $s_{i}$ 's obey the Coxeter relations

$$
\left(s_{i} s_{j}\right)^{m_{i j}}=1,
$$

where $m_{i i}=1$ and (for $i \neq j$ ) $m_{i j}=2,3,4$ or 6 , according to whether $a_{i j} a_{j i}=0,1,2$ or 3 . (If $a_{i j} a_{j i} \geqslant 4$, there is no relation of the form $\left(s_{i} s_{j}\right)^{m}=1$.)

Proposition 2.1. The operators $\left\{S_{i}(b, c): i \in I\right\}$ satisfy the Coxeter relations.

Proof. It is straightforward to check that

$$
S_{i}(0, b) S_{i}(0, c)=S_{i}(0, c) S_{i}(0, b)=S_{i}(b, c),
$$

so we may reduce to the case $b=0$. Setting $S_{i}=S_{i}(0, c)$, we have

$$
\left(S_{i} x\right)_{j}(t)=x_{j}(t)-a_{i j} x_{i}(t)=\left(s_{i} x(t)\right)_{j} \quad(\text { if } t \leqslant c),
$$

so the Coxeter relations certainly hold for $t$ in this range. On the other hand, an easy induction shows that for each Weyl group element $w=s_{i_{1}} \cdots s_{i_{l}}$ and all $t \geqslant c$, we have

$$
\left(S_{i_{1}} \cdots S_{i_{l}} x\right)_{j}(t)=x_{j}(t)-x_{j}(c)+(w x(c))_{j},
$$

so every relation satisfied by $\left\{s_{i}: i \in I\right\}$ is also satisfied by the operators $\left\{S_{i}: i \in\right.$ $I\}$.

If we adopt the convention that $S_{i}(b, c):=S_{i}(c, b)$ for $b>c$, then $(2.1)$ is still valid in this more general sense, and

$$
S_{i}(a, b) S_{i}(b, c)=S_{i}(0, a) S_{i}(0, b)^{2} S_{i}(0, c)=S_{i}(a, c),
$$

regardless of the relative order of $a, b, c$. Another useful relation is

$$
S_{i}(b, c) S_{j}\left(b^{\prime}, c^{\prime}\right)=S_{j}\left(b^{\prime}, c^{\prime}\right) S_{i}(b, c),
$$

provided that the subintervals $[b, c]$ and $\left[b^{\prime}, c^{\prime}\right]$ have disjoint interiors. We leave the proof as an exercise.

We now define (partial) operators $E_{i}$ and $F_{i}$ on $\mathcal{C}^{I}$ by setting

$$
\begin{array}{ll}
E_{i} x:=S_{i}(b, c) x & \text { if } \delta(x, i) \leqslant-1, \\
F_{i} x:=S_{i}\left(b^{\prime}, c^{\prime}\right) x & \text { if } \varepsilon(x, i) \geqslant 1,
\end{array}
$$

where

$$
\begin{aligned}
c & =\min \left\{t \in[0,1]: x_{i}(t)=\delta(x, i)\right\}, \\
b & =\max \left\{t \in[0, c]: x_{i}(t)=\delta(x, i)+1\right\}, \\
b^{\prime} & =\max \left\{t \in[0,1]: x_{i}(1)-x_{i}(t)=\varepsilon(x, i)\right\}, \\
c^{\prime} & =\min \left\{t \in\left[b^{\prime}, 1\right]: x_{i}(1)-x_{i}(t)=\varepsilon(x, i)-1\right\} .
\end{aligned}
$$

We call $[b, c]$ and $\left[b^{\prime}, c^{\prime}\right]$ the active regions for the actions of $E_{i}$ and $F_{i}$ on $x$, respectively.

In general, these operators need not be inverses of each other. On the other hand, there is a duality between $E_{i}$ and $F_{i}$ provided by the involution $x \mapsto x^{*}$ on $\mathcal{C}^{I}$ defined by

$$
x^{*}(t):=x(1-t)-x(1) .
$$


This involution interchanges $(\delta, \varepsilon)$ with $(-\varepsilon,-\delta)$, and intertwines $E_{i}$ and $F_{i}$; i.e.,

$$
E_{i}\left(x^{*}\right)=F_{i}(x)^{*} .
$$

Definition 2.2. A subset $X$ of $\mathcal{C}^{I}$ is A-integrable if for all $i \in I$,

(i) $\delta(x, i) \in \mathbf{Z}$ and $\varepsilon(x, i) \in \mathbf{Z}$ for all $x \in X$, and

(ii) $X$ is closed under the action of $E_{i}$ and $F_{i}$.

The existence of (nontrivial) $A$-integrable sets is not obvious. Indeed, the operators $E_{i}$ and $F_{i}$ need not preserve the integrality of $\delta$ and $\varepsilon$, so there need not exist an $A$-integrable set containing a particular continuous map $x$, even if it satisfies (i).

Note that the dual $X^{*}$ of an $A$-integrable set $X$ is also $A$-integrable, so any property of (say) the operators $E_{i}$ valid for all $A$-integrable sets is also a property of the $F_{i}$ 's.

Lemma 2.3. Let $X$ be an $A$-integrable set, and assume $x \in X$ is in the domain of $E_{i}$.

(a) If $i \neq j$, then $x_{j}(t) \geqslant\left(E_{i} x\right)_{j}(t) \geqslant x_{j}(t)+a_{i j}$ for all $t$.

(b) If $[b, c]$ is the active region for $E_{i}$ on $x$, then $x_{i}(t) \geqslant \delta(x, i)+1$ for all $t \leqslant b$.

(c) We have $\Delta_{i} \delta(x, i)=\Delta_{i} \varepsilon(x, i)=1$. In particular, $\delta\left(E_{i} x, i\right)=\delta(x, i)+1$.

(d) $E_{i}$ and $F_{i}$ are inverses on $X$.

Proof. (a) Letting $[b, c]$ denote the active region for $E_{i}$ on $x$, we have

$$
\left(E_{i} x\right)_{j}(t)=\left\{\begin{array}{cl}
x_{j}(t) & \text { if } 0 \leqslant t \leqslant b \\
x_{j}(t)-a_{i j}\left(x_{i}(t)-(\delta(x, i)+1)\right) & \text { if } b \leqslant t \leqslant c \\
x_{j}(t)+a_{i j} & \text { if } c \leqslant t \leqslant 1
\end{array}\right.
$$

Now use the fact that $a_{i j} \leqslant 0$ and $\delta(x, i)<x_{i}(t)<\delta(x, i)+1$ for $b<t<c$.

(b) Bearing in mind that (2.4) is valid even for $i=j$, we have

$$
\begin{array}{cl}
\left(E_{i} x\right)_{i}(t) \geqslant \delta(x, i)+2 & \text { if } t \geqslant c, \\
\left(E_{i} x\right)_{i}(t)=2(\delta(x, i)+1)-x_{i}(t) \geqslant \delta(x, i)+1 & \text { if } b \leqslant t \leqslant c,
\end{array}
$$

and equality occurs in the second case at $t=b$. Hence, $\left(E_{i} x\right)_{i}(t)$ achieves its minimum at some point $t \leqslant b$. Now since $\left(E_{i} x\right)_{i}(t)=x_{i}(t)$ for $t \leqslant b$, if there were some $t \leqslant b$ for which $x_{i}(t)<\delta(x, i)+1$, then $\delta\left(E_{i} x, i\right)$ would be an integer $<\delta(x, i)+1$, hence $\leqslant \delta(x, i)$, contradicting the fact that $x_{i}(t)>\delta(x, i)$ for all $t<c$.

(c) The above reasoning shows that the minimum of $\left(E_{i} x\right)_{i}(t)$ is achieved at $t=b$, where we have $\left(E_{i} x\right)(b)=\delta(x, i)+1$. Hence, $\delta\left(E_{i} x, i\right)=\delta(x, i)+1, \Delta_{i} \delta(x, i)=1$, and $\varepsilon\left(E_{i} x, i\right)=\left(E_{i} x\right)_{i}(1)-\delta\left(E_{i} x, i\right)=\left(x_{i}(1)+2\right)-(\delta(x, i)+1)=\varepsilon(x, i)+1$.

(d) Since equality occurs in (2.5) only at $t=b$, it follows that $b$ is the largest value of $t$ for which $\left(E_{i} x\right)_{i}(t)=\delta\left(E_{i} x, i\right)$ (or equivalently, $\left(E_{i} x\right)_{i}(1)-\left(E_{i} x\right)_{i}(t)=$ $\left.\varepsilon\left(E_{i} x, i\right)\right)$. Similarly, since $x_{i}(t)>\delta(x, i)$ for $t<c$, it follows that $t=c$ is the smallest $t \geqslant b$ for which $\left(E_{i} x\right)_{i}(t)=\delta\left(E_{i} x, i\right)+1$. This shows that $[b, c]$ is the active region for $F_{i}$ on $E_{i} x$, and hence $F_{i} E_{i} x=S_{i}(b, c)^{2} x=x$ by Proposition 2.1. Dually, it follows that $E_{i} F_{i} x=x$ for all $x \in X$ in the domain of $F_{i}$, so $E_{i}$ and $F_{i}$ form an inverse pair.

Now that $E_{i}$ and $F_{i}$ are known to be inverses on $X$, one may encode their actions by directing an edge with color $i$ from $y$ to $x$ whenever $y=E_{i} x$. In this way, every $A$-integrable set may be viewed as an edge-colored directed graph. 
Theorem 2.4. For all generalized Cartan matrices $A$, the graph of every A-integrable set is $A$-regular (i.e., satisfies axioms (P1)-(P6) and $\left.\left(P 5^{\prime}\right)-\left(P 6^{\prime}\right)\right)$.

Proof. Lemma 2.3(c) and its dual show that every $i$-monochromatic directed path through $x$ has length at most $\varepsilon(x, i)-\delta(x, i)$, whence (P1) holds. Furthermore, (P2) is essentially a reformulation of Lemma $2.3(\mathrm{~d})$. It should also be noted that this lemma shows that the definitions of $E_{i}, F_{i}, \delta$ and $\varepsilon$, we are using here are compatible with the graph-theoretic formulations of Section 1.

To verify $(\mathrm{P} 3)$, recall that $x_{j}(1)=\delta(x, j)+\varepsilon(x, j)$, whence

$$
\Delta_{i} \delta(x, j)+\Delta_{i} \varepsilon(x, j)=\left(E_{i} x\right)_{j}(1)-x_{j}(1)=a_{i j} .
$$

For (P4), recall that $\left(E_{i} x\right)_{j}(t) \leqslant x_{j}(t)$ for $i \neq j$ and all $t$ (Lemma 2.3(a)), whence $\delta\left(E_{i} x, j\right) \leqslant \delta(x, j)$ and $\Delta_{i} \delta(x, j) \leqslant 0$. Duality yields $\Delta_{i} \varepsilon(x, j) \leqslant 0$.

Lemma 2.5. If $i \neq j$ and the respective active regions for $E_{i}$ and $E_{j}$ on $x$ are $\left[b_{i}, c_{i}\right]$ and $\left[b_{j}, c_{j}\right]$, then $\Delta_{i} \delta(x, j)=a_{i j}$ (and hence $\Delta_{i} \varepsilon(x, j)=0$ by (P3)) unless $c_{j} \leqslant b_{i}$; i.e., the active region for $E_{i}$ must lie to the right of the active region for $E_{j}$.

Proof. Let $b_{j}^{\prime}=\max \left\{t \in[0,1]: x_{j}(t)=\delta(x, j)\right\}$. Since $\left(E_{i} x\right)_{j}(t)=x_{j}(t)+a_{i j}$ for $t \geqslant c_{i}$, it follows that if $b_{j}^{\prime} \geqslant c_{i}$, then $\left(E_{i} x\right)_{j}\left(b_{j}^{\prime}\right)=\delta(x, j)+a_{i j}$, and hence $\Delta_{i} \delta(x, j) \leqslant a_{i j}$. On the other hand, (P3) and (P4) imply $\Delta_{i} \delta(x, j) \geqslant a_{i j}$, so equality occurs.

Note also that if $a_{i j}=0$, then $\left(E_{i} x\right)_{j}=x_{j}$ and $\Delta_{i} \delta(x, j)=a_{i j}=0$.

Thus we may assume $b_{j}^{\prime}<c_{i}$ and $a_{i j}<0$. In particular, $b_{j}^{\prime}<1$, so the last occasion when $x_{j}(1)-x_{j}(t)$ achieves its maximum value (or equivalently, $x_{j}(t)$ achieves its minimum) is not at $t=1$, so this maximum is positive. Hence $\varepsilon(x, j)>$ 0 and $F_{j} x$ is defined. The active region for $F_{j}$ on $x$ is therefore $\left[b_{j}^{\prime}, c_{j}^{\prime}\right]$ for some $c_{j}^{\prime}>b_{j}^{\prime}$. Now since $a_{i j}<0$, we have $\left(F_{j} x\right)_{i}(t) \geqslant x_{i}(t)$ for all $t$, with strict inequality for $t>b_{j}^{\prime}$. Since this range includes $c_{i}$ and all subsequent values for $t$ where $x_{i}(t)$ achieves its minimum value, it follows that $\delta\left(F_{j} x, i\right)>\delta(x, i)$. On the other hand, we have $c_{j} \leqslant b_{j}^{\prime}$; therefore, $x_{i}\left(c_{j}\right)=\left(F_{j} x\right)_{i}\left(c_{j}\right) \geqslant \delta\left(F_{j} x, i\right) \geqslant \delta(x, i)+1$. However, $b_{i}=\max \left\{t \in\left[0, c_{i}\right]: x_{i}(t) \geqslant \delta(x, i)+1\right\}$, so $c_{j} \leqslant b_{i}$.

Proof of (P5) and $\left(\mathrm{P}^{\prime}\right)$. These two properties are equivalent by duality, so it suffices to prove (P5). Assuming that $x \in X$ and $i, j \in I$ satisfy the hypothesis of (P5), let $\left[b_{i}, c_{i}\right]$ and $\left[b_{j}, c_{j}\right]$ denote the active regions for $E_{i}$ and $E_{j}$ on $x$. We claim that $\left[b_{i}, c_{i}\right]$ and $\left[b_{j}, c_{j}\right]$ are also the active regions for $E_{i}$ on $E_{j} x$ and $E_{j}$ on $E_{i} x$, respectively.

This is clear when $a_{i j}=0$, since $\left(E_{i} x\right)_{j}=x_{j}$ and $\left(E_{j} x\right)_{i}=x_{i}$ in that case. Otherwise, we have $a_{i j}<0$ and $\Delta_{i} \delta(x, j)=0 \neq a_{i j}$, so Lemma 2.5 implies $c_{j} \leqslant$ $b_{i}$. Since $\left(E_{i} x\right)_{j}(t)=x_{j}(t)$ for $t \leqslant b_{i}$, it follows that $c_{j}$ is the first value of $t$ where $\left(E_{i} x\right)_{j}(t)=\delta(x, j)=\delta\left(E_{i} x, j\right)$, so $\left[b_{j}, c_{j}\right]$ is the active region for $E_{j}$ on $E_{i} x$. Similarly, we have $\left(E_{j} x\right)_{i}(t)=x_{i}(t)+a_{j i}$ for all $t \geqslant c_{j}$ (and hence for $b_{i} \leqslant t \leqslant c_{i}$ ), so

$$
\delta\left(E_{j} x, i\right)=\delta(x, i)+a_{j i}
$$

and $\left[b_{i}, c_{i}\right]$ is the active region for $E_{i}$ on $E_{j} x$. Note that (2.6) is valid even if $a_{i j}=0$.

Now that the claim is established, one may use (2.3) to deduce that

$$
E_{j} E_{i} x=S_{j}\left(b_{j}, c_{j}\right) S_{i}\left(b_{i}, c_{i}\right) x=S_{i}\left(b_{i}, c_{i}\right) S_{j}\left(b_{j}, c_{j}\right) x=E_{i} E_{j} x .
$$


To complete the proof, we need to confirm that $\nabla_{j} \varepsilon(y, i)=0$, where $y=E_{i} E_{j} x=$ $E_{j} E_{i} x$. Applying Lemma 2.3(c) and (2.6), we obtain

$$
\nabla_{j} \delta(y, i)=\delta\left(E_{i} E_{j} x, i\right)-\delta\left(E_{i} x, i\right)=\left(\delta\left(E_{j} x, i\right)+1\right)-(\delta(x, i)+1)=a_{j i},
$$

and this is equivalent to $\nabla_{j} \varepsilon(y, i)=0$ by (P3).

Lemma 2.6. If $E_{i} x$ and $E_{j} x$ are defined and $\Delta_{i} \delta(x, j)=\Delta_{j} \delta(x, i)=-1$, then

(a) $\delta\left(E_{i} E_{j} x, j\right)=\delta(x, j)+1$, and

(b) $\delta\left(E_{i}^{2} E_{j} x, j\right)=\delta(x, j)+1+a_{i j}$.

Proof. We have $\delta\left(E_{j} x, i\right)=\delta(x, i)-1 \leqslant-2$, so $E_{i}^{2} E_{j} x$ (and similarly $E_{j}^{2} E_{i} x$ ) is defined. Let $\left[b_{i}, c_{i}\right]$ and $\left[b_{i}^{\prime}, c_{i}^{\prime}\right]$ denote the active regions for $E_{i}$ acting on $x$ and $E_{j} x$, and similarly define $\left[b_{j}, c_{j}\right]$ and $\left[b_{j}^{\prime}, c_{j}^{\prime}\right]$. We must have $b_{j}<c_{i}^{\prime}$, since the minimum value for $\left(E_{j} x\right)_{i}(t)$ occurs at a point $t$ where $\left(E_{j} x\right)_{i}(t)<\delta(x, i) \leqslant x_{i}(t)$; likewise, $b_{i}<c_{j}^{\prime}$.

Since (P4) implies $\delta\left(E_{i} E_{j} x, j\right) \leqslant \delta\left(E_{j} x, j\right)=\delta(x, j)+1$, it suffices for (a) to show that $\left(E_{i} E_{j} x\right)_{j}(t)>\delta(x, j)$ for all $t$. For this, note that $\left(E_{i} E_{j} x\right)_{j}(t)=\left(E_{j} x\right)_{j}(t) \geqslant$ $\delta(x, j)+1$ for $t \leqslant b_{i}^{\prime}$. Furthermore, if $a_{i j}=-1$ and $t \geqslant b_{i}^{\prime}$, then

$$
\left(E_{i} E_{j} x\right)_{j}(t) \geqslant\left(E_{j} x\right)_{j}(t)-1 \geqslant \delta(x, j) .
$$

The first of these inequalities is strict for $t<c_{i}^{\prime}$, and the second is strict for $t>$ $b_{j}$; since $b_{j}<c_{i}^{\prime}$, this covers all $t \geqslant b_{i}^{\prime}$. Otherwise, if we have $a_{i j} \neq-1$, then $\Delta_{i} \delta(x, j) \neq a_{i j}$, whence Lemma 2.5 implies $c_{j} \leqslant b_{i}$ and therefore $\left[b_{i}^{\prime}, c_{i}^{\prime}\right]=\left[b_{i}, c_{i}\right]$. Furthermore, for $t \geqslant c_{j}$ (a range that includes all $t>b_{i}^{\prime}$ ), we have

$$
\left(E_{i} E_{j} x\right)_{j}(t)=\left(E_{i} x\right)_{j}(t)+2 \geqslant \delta\left(E_{i} x, j\right)+2=\delta(x, j)+1 .
$$

For (b), (P3) and (P4) imply $\delta\left(E_{i}^{2} E_{j} x, j\right) \geqslant \delta\left(E_{i} E_{j} x, j\right)+a_{i j}=\delta(x, j)+1+a_{i j}$; so it suffices to show that $\left(E_{i}^{2} E_{j} x\right)_{j}(t)<\delta(x, j)+2+a_{i j}$ for some $t$. Since $\left(E_{j} x\right)_{i}\left(b_{i}^{\prime}\right)=$ $\delta(x, i)$ and $\left(E_{j} x\right)_{i}\left(c_{i}\right) \leqslant x_{i}\left(c_{i}\right)=\delta(x, i)$, it follows that the active region for $E_{i}$ on $E_{i} E_{j} x$ lies to the left of both $b_{i}^{\prime}$ and $c_{i}$. Hence, $\left(E_{i}^{2} E_{j} x\right)_{j}(t)=\left(E_{i} E_{j} x\right)_{j}(t)+a_{i j}$ for all $t \geqslant \min \left(b_{i}^{\prime}, c_{i}\right)$. Therefore, if $c_{j}>\min \left(b_{i}^{\prime}, c_{i}\right)$, we may select $t \geqslant \min \left(b_{i}^{\prime}, c_{i}\right)$ so that $b_{j}<t<c_{j}$, whence

$$
\left(E_{i}^{2} E_{j} x\right)_{j}(t)=\left(E_{i} E_{j} x\right)_{j}(t)+a_{i j} \leqslant\left(E_{j} x\right)_{j}(t)+a_{i j}<\delta(x, j)+2+a_{i j} .
$$

The other possibility is that $c_{j} \leqslant b_{i}^{\prime}$ and $c_{j} \leqslant c_{i}$. However $\left(E_{j} x\right)_{i}(t) \geqslant x_{i}(t)+a_{j i}$, with equality if $t \geqslant c_{j}$. Since this range includes $c_{i}$ and $b_{i}^{\prime}$, it follows that $\left[b_{i}, c_{i}\right]=$ $\left[b_{i}^{\prime}, c_{i}^{\prime}\right]$. Recalling that $c_{j}^{\prime}>b_{i}$, we therefore have $c_{j}^{\prime}>b_{i}=b_{i}^{\prime} \geqslant c_{j}$, and hence

$$
\left(E_{i}^{2} E_{j}\right)_{j}\left(c_{j}^{\prime}\right)=\left(E_{i} E_{j} x\right)_{j}\left(c_{j}^{\prime}\right)+a_{i j}=\left(E_{i} x\right)_{j}\left(c_{j}^{\prime}\right)+2+a_{i j}=\delta(x, j)+1+a_{i j} .
$$

Proof of (P6) and $\left(\mathrm{P} 6^{\prime}\right)$. These two properties are dual, so it suffices to prove (P6). If $x \in X$ and $i, j \in I$ satisfy the hypothesis of (P6), Lemma 2.6 implies $\delta\left(E_{i}^{2} E_{j} x, j\right)$ $<0$ and (by symmetry) $\delta\left(E_{j}^{2} E_{i} x, i\right)<0$, so $E_{j} E_{i}^{2} E_{j} x$ and $E_{i} E_{j}^{2} E_{i} x$ are defined. If we assume temporarily that these two expressions are equal, say $y=E_{i} E_{j}^{2} E_{i} x=$ $E_{j} E_{i}^{2} E_{j} x$, then Lemma 2.6 also implies

$$
\begin{aligned}
\nabla_{i} \delta(y, j) & =\delta\left(E_{j} E_{i}^{2} E_{j} x, j\right)-\delta\left(E_{j}^{2} E_{i} x, j\right)=\left(\delta\left(E_{i}^{2} E_{j} x, j\right)+1\right)-\left(\delta\left(E_{i} x, j\right)+2\right) \\
& =\left(\delta(x, j)+2+a_{i j}\right)-(\delta(x, j)+1)=1+a_{i j} .
\end{aligned}
$$

This is equivalent to $\nabla_{i} \varepsilon(y, j)=-1$ by (P3); one similarly obtains $\nabla_{j} \varepsilon(y, i)=-1$.

The remaining task is therefore to show that $E_{i} E_{j}^{2} E_{i} x=E_{j} E_{i}^{2} E_{j} x$. For this, let $\left[b_{i}, c_{i}\right]$ and $\left[b_{j}, c_{j}\right]$ denote the active regions for $E_{i}$ and $E_{j}$ on $x$. 
Case 1: $c_{j} \leqslant b_{i}$ or $c_{i} \leqslant b_{j}$ (i.e., the active regions have disjoint interiors). By symmetry, we may assume $c_{j} \leqslant b_{i}$, and hence $b_{j}<c_{i}$. It follows that $a_{j i}=$ $\Delta_{j} \delta(x, i)=-1$ by Lemma 2.5 , and the active region for $E_{i}$ on $E_{j} x$ must be $\left[b_{i}, c_{i}\right]$. Furthermore, since $\delta\left(E_{i} E_{j} x, i\right)=\delta(x, i)$ and $E_{i} E_{j} x(t)=E_{j} x(t)$ for $t \leqslant b_{i}$, the active region for $E_{i}$ on $E_{i} E_{j} x$ must be $\left[b_{i}^{\prime}, c_{i}^{\prime}\right]$, where

$$
\begin{aligned}
c_{i}^{\prime} & =\min \left\{t \in[0,1]:\left(E_{j} x\right)_{i}(t)=\delta(x, i)\right\}, \\
b_{i}^{\prime} & =\max \left\{t \in\left[0, c_{i}^{\prime}\right]:\left(E_{j} x\right)_{i}(t)=\delta(x, i)+1\right\} .
\end{aligned}
$$

In particular, it should be noted that $c_{i}^{\prime} \leqslant b_{i}<c_{i}$, since $\left(E_{j} x\right)_{i}\left(b_{i}\right)=x_{i}\left(b_{i}\right)+a_{j i}=$ $\delta(x, i)$. We also must have $b_{j}<c_{i}^{\prime}$; otherwise, $x_{i}\left(c_{i}^{\prime}\right)=\left(E_{j} x\right)_{i}\left(c_{i}^{\prime}\right)=\delta(x, i)$ and we contradict the fact that $c_{i}^{\prime}<c_{i}$.

Similarly, the active region for $E_{j}$ on $E_{i} x$ is $\left[b_{j}^{\prime}, c_{j}^{\prime}\right]$, where

$$
\begin{aligned}
c_{j}^{\prime} & =\min \left\{t \in[0,1]:\left(E_{i} x\right)_{j}(t)=\delta(x, j)-1\right\}, \\
b_{j}^{\prime} & =\max \left\{t \in\left[0, c_{j}^{\prime}\right]:\left(E_{i} x\right)_{j}(t)=\delta(x, j)\right\} .
\end{aligned}
$$

We must have $b_{i} \leqslant b_{j}^{\prime}$; otherwise, there would exist $t \leqslant b_{i}$ such that $b_{j}^{\prime}<t<c_{j}^{\prime}$ and $x_{j}(t)=\left(E_{i} x\right)_{j}(t)<\delta(x, j)$. Therefore, since $\delta\left(E_{j} E_{i} x, j\right)=\delta(x, j)$ and $E_{j} E_{i} x(t)=$ $x(t)$ for $t \leqslant b_{j}^{\prime}$ (a range that includes $\left[b_{j}, c_{j}\right]$ ), the active region for $E_{j}$ on $E_{j} E_{i} x$ must be $\left[b_{j}, c_{j}\right]$.

We have $\delta\left(E_{j}^{2} E_{i} x, i\right)=\delta(x, i)$ (by Lemma 2.6) and $\left(E_{j}^{2} E_{i} x\right)_{i}(t)=\left(E_{j} x\right)_{i}(t)$ for $t \leqslant b_{i}$ (since $\left.b_{i} \leqslant b_{j}^{\prime}\right)$, so $\left(E_{j}^{2} E_{i} x\right)_{i}(t)$ achieves the minimum value $\delta(x, i)$ at $t=c_{i}^{\prime}$, and the active region for $E_{i}$ on $E_{j}^{2} E_{i} x$ must be $\left[b_{i}^{\prime}, c_{i}^{\prime}\right]$.

Similarly, $\delta\left(E_{i}^{2} E_{j} x, j\right)=\delta(x, j)+1+a_{i j}$ (Lemma 2.6), and for $t \leqslant b_{i}$, we have

$$
\left(E_{i}^{2} E_{j} x\right)_{j}(t) \geqslant\left(E_{i} E_{j} x\right)_{j}(t)+a_{i j}=\left(E_{j} x\right)_{j}(t)+a_{i j} \geqslant \delta(x, j)+1+a_{i j}
$$

In particular, the first inequality is strict for $t<c_{i}^{\prime}$; the second is strict for $t>b_{j}$. Recalling that $b_{j}<c_{i}^{\prime}$, we see that $\left(E_{i}^{2} E_{j} x\right)_{j}(t)$ cannot achieve its minimum value in $\left[0, b_{i}\right]$. On the other hand, for $t \geqslant b_{i}$, we have $\left(E_{i}^{2} E_{j} x\right)_{j}(t)=\left(E_{i} x\right)_{j}(t)+2+a_{i j}$, so the minimum first occurs at $t=c_{j}^{\prime}$, and the active region for $E_{j}$ on $E_{i}^{2} E_{j} x$ is $\left[b_{j}^{\prime}, c_{j}^{\prime}\right]$.

Combining these observations, we deduce that

$$
\begin{aligned}
E_{j} E_{i}^{2} E_{j} x & =S_{j}\left(b_{j}^{\prime}, c_{j}^{\prime}\right) S_{i}\left(b_{i}^{\prime}, c_{i}^{\prime}\right) S_{i}\left(b_{i}, c_{i}\right) S_{j}\left(b_{j}, c_{j}\right) x, \\
E_{i} E_{j}^{2} E_{i} x & =S_{i}\left(b_{i}^{\prime}, c_{i}^{\prime}\right) S_{j}\left(b_{j}, c_{j}\right) S_{j}\left(b_{j}^{\prime}, c_{j}^{\prime}\right) S_{i}\left(b_{i}, c_{i}\right) x .
\end{aligned}
$$

Bearing in mind that the intervals $\left[b_{j}, c_{j}\right]$ and $\left[b_{i}^{\prime}, c_{i}^{\prime}\right]$ are both to the left of $\left[b_{i}, c_{i}\right]$ and $\left[b_{j}^{\prime}, c_{j}^{\prime}\right]$, three applications of (2.3) show that these two expressions are equal.

Case 2: $b_{i}<c_{j}$ and $b_{j}<c_{i}$ (i.e., the active regions overlap). We have $b^{*}<c_{*}$, where

$$
b_{*}=\min \left(b_{i}, b_{j}\right), \quad b^{*}=\max \left(b_{i}, b_{j}\right), \quad c_{*}=\min \left(c_{i}, c_{j}\right), \quad c^{*}=\max \left(c_{i}, c_{j}\right) .
$$

Furthermore, since $x_{i}(t) \geqslant \delta(x, i)+1$ for $t \leqslant b_{i}, \delta(x, i)<x_{i}(t)<\delta(x, i)+1$ for $b_{i}<t<c_{i}$, and $x_{i}\left(c_{i}\right)=\delta(x, i)$ (and similar relations hold for $x_{j}(t)$ ), the quantity

$$
u(t):=\left(x_{i}(t)-\delta(x, i)\right)+\left(x_{j}(t)-\delta(x, j)\right)
$$


satisfies $u(t) \geqslant 2$ for $t \leqslant b_{*}, u(t)>1$ for $t \leqslant b^{*}, u(t)<2$ for $b^{*}<t<c_{*}$, and $u\left(c_{*}\right)<1$. It follows that there exist times $t_{0}, t_{1}, t_{2}$ such that

$$
\begin{aligned}
& t_{0}=\max \left\{t \in\left[0, c_{*}\right]: x_{i}(t)+x_{j}(t)=\delta(x, i)+\delta(x, j)+2\right\}, \\
& t_{1}=\min \left\{t \in[0,1]: x_{i}(t)+x_{j}(t)=\delta(x, i)+\delta(x, j)+1\right\}, \\
& t_{2}=\max \left\{t \in\left[0, c_{*}\right]: x_{i}(t)+x_{j}(t)=\delta(x, i)+\delta(x, j)+1\right\},
\end{aligned}
$$

and furthermore, $b_{*} \leqslant t_{0} \leqslant b^{*}<t_{1} \leqslant t_{2}<c_{*}$.

Since $\delta\left(E_{j} x, i\right)=\delta(x, i)-1$, the right endpoint of the active region for $E_{i}$ on $E_{j} x$ is

$$
c_{i}^{\prime}:=\min \left\{t \in[0,1]:\left(E_{j} x\right)_{i}(t)=\delta(x, i)-1\right\} .
$$

Similarly, let $c_{j}^{\prime}$ denote the right endpoint for the action of $E_{j}$ on $E_{i} x$. Since $b^{*}<c_{*}$, Lemma 2.5 implies $a_{i j}=a_{j i}=-1$. Hence $\left(E_{j} x\right)_{i}(t) \geqslant x_{i}(t)-1$, with equality only for $t \geqslant c_{j}$, so we must have $c_{i}^{\prime} \geqslant c_{j}$. If it happens that $c_{i} \geqslant c_{j}$, then we must have $c_{i}^{\prime}=c_{i}$. (By symmetry, it follows that we always have $c_{i}^{\prime}=c_{i}$ or $c_{j}^{\prime}=c_{j}$.)

Note that for $b^{*} \leqslant t \leqslant c_{*}$, we have $\left(E_{j} x\right)_{i}(t)=x_{i}(t)+x_{j}(t)-(\delta(x, j)+1)$. It follows that $\left(E_{j} x\right)_{i}\left(c_{*}\right)<\delta(x, i)$ (recall that $u\left(c_{*}\right)<1$ ), so the active region for $E_{i}$ on $E_{j} x$ has a left endpoint $\leqslant c_{*}$, and hence the active region must be $\left[t_{2}, c_{i}^{\prime}\right]$.

For $t \leqslant b_{*}$, we have $\left(E_{i} E_{j} x\right)_{i}(t)=x_{i}(t) \geqslant \delta(x, i)+1$, so the active region for $E_{i}$ on $E_{i} E_{j} x$ occurs to the right of $b_{*}$. Furthermore, we have

$$
\left(E_{i} E_{j} x\right)_{i}(t)=\left\{\begin{array}{cl}
x_{i}(t) & \text { if } t \leqslant b_{j}, \\
x_{i}(t)+x_{j}(t)-(\delta(x, j)+1) & \text { if } b_{j} \leqslant t \leqslant t_{2},
\end{array}\right.
$$

so the active region for $E_{i}$ on $E_{i} E_{j} x$ is either $\left[t_{0}, t_{1}\right]$ (if $b_{j} \leqslant b_{i}$ ) or $\left[b_{i}, t_{1}\right]\left(\right.$ if $b_{i} \leqslant b_{j}$ ).

To determine the active region for $E_{j}$ on $E_{i}^{2} E_{j} x$, recall that $a_{i j}=-1$, hence Lemma 2.6 implies $\delta\left(E_{i}^{2} E_{j} x, j\right)=\delta(x, j)$. Furthermore, since $\delta\left(E_{i} E_{j} x, j\right)=\delta(x, j)$ +1 (Lemma 2.6 again), we have $\left(E_{i}^{2} E_{j} x\right)_{j}(t) \geqslant\left(E_{i} E_{j} x\right)_{j}(t)-1 \geqslant \delta(x, j)$, with equality only for $t \geqslant t_{1}$. For $t_{1} \leqslant t \leqslant t_{2}$, we have $\left(E_{i}^{2} E_{j} x\right)_{j}(t)=\left(E_{j} x\right)_{j}(t)-1>$ $\delta(x, j)$, and for $t_{2} \leqslant t \leqslant c_{*}$, we have

$$
\begin{aligned}
& \left(E_{i}^{2} E_{j} x\right)_{j}(t)=\left(E_{i} E_{j} x\right)_{j}(t)-1=\left(E_{j} x\right)_{j}(t)+\left(E_{j} x\right)_{i}(t)-\left(\delta\left(E_{j} x, i\right)+1\right)-1 \\
& \quad=\left(2(\delta(x, j)+1)-x_{j}(t)\right)+\left(x_{i}(t)+x_{j}(t)-(\delta(x, j)+1)\right)-(\delta(x, i)+1) \\
& \quad=x_{i}(t)+\delta(x, j)-\delta(x, i) .
\end{aligned}
$$

Thus, if $c_{i} \leqslant c_{j}$, then $\left(E_{i}^{2} E_{j} x\right)_{j}(t)$ first achieves the minimum value $\delta(x, j)$ at $t=c_{i}$. Otherwise, if $c_{j} \leqslant c_{i}$, then we have $x_{i}(t)>\delta(x, i)$ and $\left(E_{i}^{2} E_{j} x\right)_{j}(t)>\delta(x, j)$ for $t<c_{*}$, so $\left(E_{i}^{2} E_{j} x\right)_{j}(t)=\left(E_{i} x\right)_{j}(t)+2+a_{i j}=\left(E_{i} x\right)_{j}(t)+1$ for $t \geqslant c_{*}$. Hence $\left(E_{i}^{2} E_{j} x\right)_{j}(t)$ first achieves the value $\delta(x, j)$ at $t=c_{j}^{\prime}$ (by the definition of $c_{j}^{\prime}$ ). Thus the right endpoint of the active region is $c_{i}$ if $c_{i} \leqslant c_{j}$ and $c_{j}^{\prime}$ if $c_{j} \leqslant c_{i}$. Now for $b^{*} \leqslant t \leqslant t_{1}$, we have

$$
\begin{aligned}
\left(E_{i}^{2} E_{j} x\right)_{j}(t)=\left(E_{i} E_{j} x\right)_{j}(t)+\left(E_{i} E_{j} x\right)_{i}(t)-\left(\delta\left(E_{i} E_{j} x, i\right)+1\right) \\
\quad=\left(E_{j} x\right)_{j}(t)+\left(E_{j} x\right)_{i}(t)-(\delta(x, i)+1) \\
\quad=\left(2(\delta(x, j)+1)-x_{j}(t)\right)+\left(x_{i}(t)+x_{j}(t)-(\delta(x, j)+1)\right)-(\delta(x, i)+1) \\
\quad=x_{i}(t)+\delta(x, j)-\delta(x, i)
\end{aligned}
$$

and $x_{i}(t)<\delta(x, i)+1$ for $t>b_{i}$, so the left endpoint of the active region is $\leqslant b^{*}$. Furthermore, if $b_{j} \leqslant b_{i}$ then $b^{*}=b_{i}$ and $\left(E_{i}^{2} E_{j} x\right)_{j}\left(b_{i}\right)=\delta(x, j)+1$, so the left 


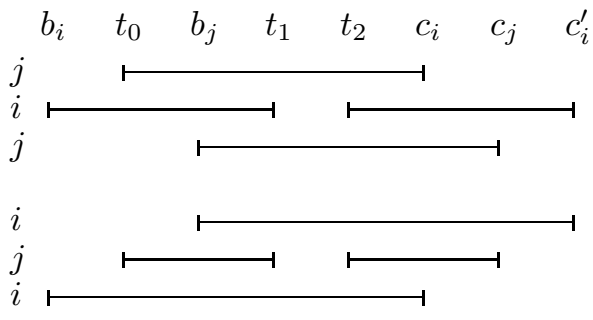

(a) $b_{i} \leqslant b_{j}, c_{i} \leqslant c_{j}$

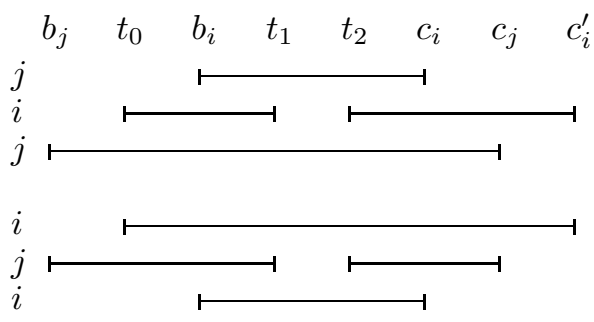

(b) $b_{j} \leqslant b_{i}, c_{i} \leqslant c_{j}$

Figure 4. Active regions for $E_{j} E_{i}^{2} E_{j}$ and $E_{i} E_{j}^{2} E_{i}$.

endpoint must be $b_{i}$. Otherwise, if $b_{i} \leqslant b_{j}$, then for $t_{0} \leqslant t \leqslant b^{*}$, we have

$$
\begin{aligned}
\left(E_{i}^{2} E_{j} x\right)_{j}(t) & =\left(E_{i} E_{j} x\right)_{j}(t)+\left(E_{i} E_{j} x\right)_{i}(t)-\left(\delta\left(E_{i} E_{j} x, i\right)+1\right) \\
& =x_{j}(t)+x_{i}(t)-(\delta(x, i)+1),
\end{aligned}
$$

so the left endpoint in this case is $t_{0}$.

By symmetry, the active regions involved in the calculation of $E_{i} E_{j}^{2} E_{i} x$ can be obtained by switching the roles of $i$ and $j$ in the above analysis. In particular, note that $t_{0}, t_{1}, t_{2}$ are unaffected by this switch. If we assume for simplicity that $c_{i} \leqslant c_{j}$ (and hence $c_{j}^{\prime}=c_{j}$ ), then we have shown

$$
\begin{gathered}
E_{j} E_{i}^{2} E_{j} x= \begin{cases}S_{j}\left(t_{0}, c_{i}\right) S_{i}\left(b_{i}, t_{1}\right) S_{i}\left(t_{2}, c_{i}^{\prime}\right) S_{j}\left(b_{j}, c_{j}\right) x & \text { if } b_{i} \leqslant b_{j}, \\
S_{j}\left(b_{i}, c_{i}\right) S_{i}\left(t_{0}, t_{1}\right) S_{i}\left(t_{2}, c_{i}^{\prime}\right) S_{j}\left(b_{j}, c_{j}\right) x & \text { if } b_{i} \geqslant b_{j},\end{cases} \\
E_{i} E_{j}^{2} E_{i} x= \begin{cases}S_{i}\left(b_{j}, c_{i}^{\prime}\right) S_{j}\left(t_{0}, t_{1}\right) S_{j}\left(t_{2}, c_{j}\right) S_{i}\left(b_{i}, c_{i}\right) x & \text { if } b_{i} \leqslant b_{j}, \\
S_{i}\left(t_{0}, c_{i}^{\prime}\right) S_{j}\left(b_{j}, t_{1}\right) S_{j}\left(t_{2}, c_{j}\right) S_{i}\left(b_{i}, c_{i}\right) x & \text { if } b_{i} \geqslant b_{j} .\end{cases}
\end{gathered}
$$

The relative positions of the various active regions are displayed in Figure 4.

To see that the two expressions are equal, decompose each reflection operator into operators whose active regions are the (internally) disjoint intervals $\left[b_{*}, t_{0}\right],\left[t_{0}, b^{*}\right]$, $\left[b^{*}, t_{1}\right],\left[t_{1}, t_{2}\right],\left[t_{2}, c_{i}\right],\left[c_{i}, c_{j}\right]$, and $\left[c_{j}, c_{i}^{\prime}\right]$ using (2.2). Since reflection operators with disjoint active regions commute, it thus suffices to check the equality of the products of operators involving each of these seven subintervals separately. Now since $a_{i j}=a_{j i}=-1$, the reflection operators $S_{i}=S_{i}(b, c)$ and $S_{j}=S_{j}(b, c)$ on any given subinterval $[b, c]$ satisfy the Coxeter relations $S_{i}^{2}=S_{j}^{2}=\left(S_{i} S_{j}\right)^{3}=1$ (Proposition 2.1), and by inspecting the illustrations in Figure 4, one can easily see that the necessary equalities are consequences of these relations. For example, for the subinterval $\left[t_{2}, c_{i}\right]$, the equality in question is the braid relation $S_{j} S_{i} S_{j}=$ $S_{i} S_{j} S_{i}$.

\section{Crystals of representations are $A$-regular posets}

By Theorem 2.4, the existence of $A$-regular posets reduces to the existence of $A$-integrable sets of paths. For the latter, one may appeal to the Path Model.

Theorem 3.1 (Littelmann [L1). If $A$ is a symmetrizable Cartan matrix, then for each nonnegative integer I-tuple $\mu=\left(\mu_{i}: i \in I\right)$, there is an A-integrable set of paths containing a maximal object $x$ with $\varepsilon(x, i)=\mu_{i}$ for all $i \in I$. (In fact, one may take $x$ to be a straight line from 0 to $\mu$.) 
We remark that verifying the above result is a relatively basic part of the Path Model, and may be deduced from a few standard facts about the Bruhat ordering. For example, see Section 8 of [St] (particularly Lemma 8.2(a) and the discussion preceding it).

Combining the above result with Theorem 2.4 and Propositions 1.2 and 1.4, we obtain

Theorem 3.2. If $A$ is simply-laced, then for each nonnegative integer I-tuple $\mu$, there is a unique A-regular poset $P(\mu)$ with a maximum vertex that has rise vector $\mu$.

It would be interesting to find a direct, graph-theoretic proof that the algorithm described in Remark 1.5 generates $A$-regular posets in all simply-laced cases. This would provide an alternative proof of Theorem 3.2 that bypasses Theorems 2.4 and 3.1.

By a theorem proved independently by Joseph [J] and Kashiwara [K3], one knows that the edge-colored directed graphs produced by the Path Model coincide with the crystal graphs of irreducible highest weight $U_{q}(A)$-representations in Kashiwara's theory [K2]. (Here, $U_{q}(A)$ denotes the quantized enveloping algebra of the KacMoody algebra with Cartan matrix $A$.) Thus we have

Theorem 3.3. If $A$ is a simply-laced Cartan matrix, then the crystal graph of the irreducible $U_{q}(A)$-module of highest weight $\mu$ is (the Hasse diagram of) $P(\mu)$.

As the crystal of an irreducible highest weight module, the vertices of $P(\mu)$ may be assigned weights so that the corresponding generating series is the Weyl character $\chi(\mu)$. In addition, the depth vectors of the vertices provide an easy rule for decomposing every Weyl character product $\chi(\mu) \chi(\nu)$ (e.g., see [L1], [L2], or [St]). It would be interesting to deduce this directly from the axioms; one possible approach would be to show that every $A$-regular directed graph has a "coherent timing pattern" in the sense of [St].

The main problem remaining is to find analogues of Theorems 3.2 and 3.3 for all symmetrizable Cartan matrices, or at least the Cartan matrices of semisimple Lie algebras. Since the work in Section 2 applies to all generalized Cartan matrices, it follows that the axioms for $A$-regularity hold without modification for the crystal graphs corresponding to all highest weight $U_{q}(A)$-modules for all symmetrizable $A$.

The missing ingredient is a stronger set of axioms describing the local structure of an $A$-integrable set in the vicinity of every triple of paths $x, E_{i} x, E_{j} x$ (for distinct $i, j \in I)$. If it happens that $\Delta_{i} \delta(x, j)=0$, or $\Delta_{j} \delta(x, i)=0$, or $\Delta_{i} \delta(x, j)=$ $\Delta_{j} \delta(x, i)=-1$, then the local structure is governed by (P5) or (P6). However, if $A$ is not simply-laced, then this does not cover all of the possibilities. Indeed, (P3) and (P4) imply $0 \geqslant \Delta_{i} \delta(x, j) \geqslant a_{i j}$ and $0 \geqslant \Delta_{j} \delta(x, i) \geqslant a_{j i}$, while Lemma 2.5 implies $\Delta_{i} \delta(x, j)=a_{i j}$ or $\Delta_{j} \delta(x, i)=a_{j i}$, leaving a total of $1-a_{i j}-a_{j i}$ possible values for the pair $\left(\Delta_{i} \delta(x, j), \Delta_{j} \delta(x, i)\right)$.

In addition to the increased number of possibilities for $\left(\Delta_{i} \delta(x, j), \Delta_{j} \delta(x, i)\right)$, a more significant complication is that this data no longer determines the local structure near $x$. For example, in Figure 5 are the crystals of three highest weight modules of type $B_{2}$ (i.e., for a $2 \times 2$ Cartan matrix $A$ with $a_{12}=-1$ and $a_{21}=-2$ ). In each graph, the vertex labeled $x$ has the property that $\left(\Delta_{1} \delta(x, 2), \Delta_{2} \delta(x, 1)\right)=$ $(-1,-2)$, but the local structures are different (e.g., the lowest vertex that can reach both $E_{1} x$ and $E_{2} x$ by a directed path is either 7,5 , or 4 steps away from $x$ ). 

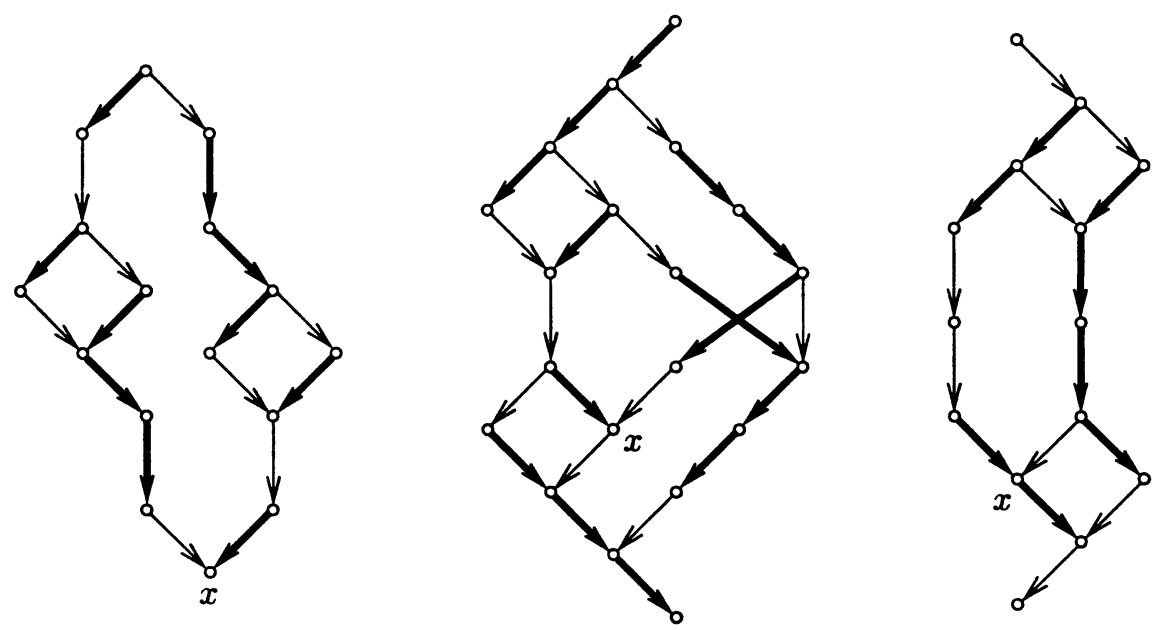

FIGURE 5. $B_{2}$-crystals with highest weights $(1,1),(3,0)$ and $(0,2)$

Computer searches suggest that these three examples represent all of the possible local behaviors; e.g., conjecturally,

$$
E_{1} E_{2}^{2} E_{1}^{3} E_{2} x=E_{2} E_{1}^{3} E_{2}^{2} E_{1} x, E_{1} E_{2} E_{1} x=E_{2} E_{1} F_{2} E_{1} E_{2} x,
$$

or

$$
E_{1} E_{2}^{2} E_{1} x=E_{2} E_{1}^{2} E_{2} x
$$

whenever $\left(\Delta_{1} \delta(x, 2), \Delta_{2} \delta(x, 1)\right)=(-1,-2)$ in a $B_{2}$-crystal. However, there are several more possibilities in $G_{2}$-crystals, even for vertices with $\left(\Delta_{1} \delta(x, 2), \Delta_{2} \delta(x, 1)\right)=$ $(-1,-2)$.

\section{REFERENCES}

[BZ] A. Berenstein and A. Zelevinsky, Tensor product multiplicities, canonical bases and totally positive varieties, Invent. Math. 143 (2001), 77-128. MR 2002c:17005

[J] A. Joseph, "Quantum groups and their primitive ideals," Springer-Verlag, New York, 1995. MR 96d:17015

[L1] P. Littelmann, A Littlewood-Richardson rule for symmetrizable Kac-Moody algebras, Invent. Math. 116 (1994), 329-346. MR 95f:17023

[L2] P. Littelmann, Paths and root operators in representation theory, Ann. of Math. 142 (1995), 499-525. MR 96m:17011

[L3] P. Littelmann, Crystal graphs and Young tableaux, J. Algebra 175 (1995), 65-87. MR 96h:17022

[L4] P. Littelmann, Cones, crystals, and patterns, Transform. Groups 3 (1998), 145-179. MR 99e:17009

[KMN] S.-J. Kang, M. Kashiwara, K. Misra, T. Miwa, T. Nakashima and A. Nakayashiki, Affine crystals and vertex models, Internat. J. Modern Phys. A 7, Supp. 1A (1992), 449-484. MR 94a:17008

[KN] M. Kashiwara and T. Nakashima, Crystal graphs for representations of the $q$-analogue of classical Lie algebras, J. Algebra 165 (1994), 295-345. MR 95c:17025

[K1] M. Kashiwara, Crystal bases of modified quantized enveloping algebra, Duke Math. J. 73 (1994), 383-413. MR 95c:17024]

[K2] M. Kashiwara, On crystal bases, in "Representations of Groups (Banff, AB, 1994)," CMS Conf. Proc. 16 (1995), 155-197. MR 97a:17016 
[K3] M. Kashiwara, Similarity of crystal bases, in "Lie algebras and their Representations (Seoul, 1995)," Contemp. Math. 194 (1996), 177-186. MR 97g:17013

[St] J. R. Stembridge, Combinatorial models for Weyl characters, Advances in Math. 168 (2002), 96-131.

Department of Mathematics, University of Michigan, Ann Arbor, Michigan 481091109 